\title{
Effects of Composite Formulation on Mechanical Properties of Biodegradable Poly(Propylene Fumarate)/Bone Fiber Scaffolds
}

\author{
Xun Zhu, Nathan Liu, Michael J. Yaszemski, and Lichun Lu \\ Departments of Orthopedic Surgery and Biomedical Engineering, Mayo Clinic College of Medicine, \\ 200 First Street SW, Rochester, MN 55905, USA \\ Correspondence should be addressed to Lichun Lu, lu.lichun@mayo.edu
}

Received 15 March 2010; Accepted 9 May 2010

Academic Editor: Shanfeng Wang

Copyright ( $\odot 2010$ Xun Zhu et al. This is an open access article distributed under the Creative Commons Attribution License, which permits unrestricted use, distribution, and reproduction in any medium, provided the original work is properly cited.

The objective of our paper was to determine the effects of composite formulation on the compressive modulus and ultimate strength of a biodegradable, in situ polymerizable poly(propylene fumarate) (PPF) and bone fiber scaffold. The following parameters were investigated: the incorporation of bone fibers (either mineralized or demineralized), PPF molecular weight, Nvinyl pyrrolidinone (NVP) crosslinker amount, benzoyl peroxide (BP) initiator amount, and sodium chloride porogen amount. Eight formulations were chosen based on a resolution III two-level fractional factorial design. The compressive modulus and ultimate strength of these formulations were measured on a materials testing machine. Absolute values for compressive modulus varied from 21.3 to $271 \mathrm{MPa}$ and 2.8 to $358 \mathrm{MPa}$ for dry and wet samples, respectively. The ultimate strength of the crosslinked composites varied from 2.1 to 20.3 MPa for dry samples and from 0.4 to $16.6 \mathrm{MPa}$ for wet samples. Main effects of each parameter on the measured property were calculated. The incorporation of mineralized bone fibers and an increase in PPF molecular weight resulted in higher compressive modulus and ultimate strength. Both mechanical properties also increased as the amount of benzoyl peroxide increased or the NVP amount decreased in the formulation. Sodium chloride had a dominating effect on the increase of mechanical properties in dry samples but showed little effects in wet samples. Demineralization of bone fibers led to a decrease in the compressive modulus and ultimate strength. Our results suggest that bone fibers are appropriate as structural enforcement components in PPF scaffolds. The desired orthopaedic PPF scaffold might be obtained by changing a variety of composite formulation parameters.

\section{Introduction}

There has been a great need for the treatment of skeletal defects which may result from tumors, trauma, or abnormal development [1]. The current methods for restoring tissue structure and function rely mostly on autograft and allograft [2-4]. Both methods, while appropriate for management of many bone defects, do have certain limitations. These include donor site morbidity after autograft harvesting and slow incorporation of cortical allograft. The other materials commonly used such as polymers, ceramics, and metals all have their associated drawbacks, such as poor integration with the host tissue, stress shielding of adjacent bone, and osteolysis from particulate wear debris [5]. The treatment regimen could be improved with the availability of a skeletal regeneration biomaterial that could be processed into the specific shape needed, provide structural support required, be replaced by new, vascularized bone tissue, and then disappear to allow the new bone to remodel along local stress lines.

Poly(propylene fumarate) (PPF) is an unsaturated linear polyester with fumarate double bonds that can be crosslinked in situ. It is biocompatible, biodegradable, osteoconductive, and capable of both preformed and injectable applications [6-9]. PPF scaffolds can be used to fill irregularly shaped defects with minimal surgical intervention. They possess mechanical properties on the order of magnitude of human trabecular bone. The fabrication of biodegradable polymer composites based on PPF for orthopaedic applications has been the subject of investigation in our laboratory [10-12]. The properties of the produced composites can be tailored for specific applications by varying different parameters 
including crosslinking density and fillers $[10,11,13,14]$. The composite formulation can include a porogen such as $\mathrm{NaCl}$ for initial porosity and a particulate ceramic such as $\beta$-tricalcium phosphate (TCP) for mechanical reinforcement and increased osteoconductivity.

Mineralized bone fibers (MBFs) are obtained by processing allograft bone. The technique involves shaving the cortical bone to produce bone fibers. MBFs have a composition identical to bone and may be used for structural reinforcement of scaffolds. They have been shown to increase the initial compressive strength of PPF/PPF-DA scaffolds [11]. The acidification of MBF produces demineralized bone fibers (DBF), which consist of nonmineral components of MBF and have accessible bone inductive factors. Demineralized bone matrices (DBMs) have extremely high-osteoinductive properties and greatly improve the integration of autogenous bone grafts in the skull [15]. In the various forms of DBM, the fiber-based grafts produced the largest new bone in a critical size cranial defect in athymic rats [16]. These grafts were found to perform as well as autografts [16]. Therefore, the incorporation of bone fibers in PPF scaffolds not only provides a structural support component but could also promote bone regeneration in bone defects.

In this study, experiments were designed to study the effects of various processing parameters, such as PPF molecular weight, incorporation of bone fibers (MBF or $\mathrm{DBF})$, crosslinker amount, initiator amount, and porogen amount, on the ultimate strength and compressive modulus of PPF/bone fiber scaffolds.

\section{Materials and Methods}

2.1. Raw Materials. Fumaryl chloride (Aldrich, Milwaukee, WI) was purified by distillation under nitrogen atmosphere. Demineralized bone fibers (DBFs) were obtained through acidification wash of mineralized bovine bone fibers (MBF) (OsteoTech Inc.). Propylene glycol, N-vinyl pyrrolidinone (NVP), N,N-dimenthyl-p-toluidene (DMT), benzoyl peroxide (BP) and sodium chloride were purchased from Aldrich Chemical (Milwaukee, WI) and used as received. All solvents were purchased from Fisher (Pittsburgh, PA) as reagent grade and used as received. Sodium chloride was sieved to obtain particles in a 106-300 $\mu \mathrm{m}$ size range and used as porogen. All experiments described below were based on a Resolution III two-level fractional factorial design varying six parameters. The six parameters included PPF molecular weight, NVP to $\mathrm{PPF}$ ratio, $\mathrm{BF}$ to polymer (PPF/NVP) ratio, $\mathrm{BF}$ type, $\mathrm{BP}$ to PPF ratio, and the percentage of $\mathrm{NaCl}$ in the composites. The experimental design and the values for all parameters are presented in Table 1

2.2. PPF Synthesis. PPF was synthesized by a two-step reaction process as described previously in [17]. Briefly, fumaryl chloride was added dropwise to a solution of propylene glycol in methylene chloride at $0^{\circ} \mathrm{C}$ under nitrogen in the presence of $\mathrm{K}_{2} \mathrm{CO}_{3}$. After addition of fumaryl chloride, the reaction mixture was stirred for an additional $2 \mathrm{~h}$ at $0^{\circ} \mathrm{C}$ before water was added to dissolve the inorganic salt. The organic phase was separated and dried over $\mathrm{Na}_{2} \mathrm{SO}_{4}$. After filtration of the mixture and evaporation of the solvent, the formed di(2-hydroxylpropyl) fumarate was converted to PPF by transesterification at $160^{\circ} \mathrm{C}$ and $0.5 \mathrm{mmHg}$. The produced polymer was purified by solution precipitation forming a viscous liquid. Gel permeation chromatography with a differential refractometer (Waters 410, Milford, MA) was used to determine polymer molecular weight distributions. A Phenogel column $(300 \times 7.8 \mathrm{~mm}, 5 \mathrm{~nm}$, mixed bed, Phenomenex, Torrance, CA) and a Phenogel guard column ( $7.8 \mathrm{~mm}, 5 \mathrm{~nm}$, mixed bed, Phenomenex) were employed for the elution of polymer solution in chloroform at flow rate of $1 \mathrm{ml} / \mathrm{min}$. Polystyrene standards were utilized to obtain a calibration curve for calculating the polymer molecular weights.

2.3. Demineralization of Bone Fibers. MBF were weighed to the nearest gram and soaked in 15 volume of a $0.6 \mathrm{~N} \mathrm{HCl}$ solution with $0.025 \%$ Triton $\mathrm{X}-100$. When the $\mathrm{pH}$ of the solution was more than 1 , the acid was decanted and replaced with another 15 volume of fresh $0.6 \mathrm{~N} \mathrm{HCl}$ without triton. $\mathrm{PH}$ readings were taken every ten minutes until it is more than 1 . The acid was carefully decanted and the same volume of distilled, deionized water $\left(\mathrm{ddH}_{2} \mathrm{O}\right)$ was added. The water wash was repeated several times until the $\mathrm{pH}$ is greater than 3. The resulting DMF were soaked in $70 \%$ ethanol for $30 \mathrm{~min}$. and collected on a $106 \mu \mathrm{m}$ sieve. DMF were washed again with $\mathrm{ddH}_{2} \mathrm{O}$ and freeze-dried for $24 \mathrm{hrs}$.

2.4. Experimental Design. All experiments described below were based on a Resolution III two-level fractional factorial design varying six parameters [18]. The experimental design and the values for all parameters are presented in Table 1. Six processing parameters were varied to determine their effects on mechanical properties of the composite scaffolds. The high $(+)$ and low $(-)$ values for all parameters are listed (Table 1(a)). The number average molecular weights $(\mathrm{Mn})$ of PPF were chosen as 4000 and 2000. The ratios of NVP to PPF were 0.7 and $0.5 \mathrm{ml}$ per gram. The weight percentages of bone fibers to polymer were $20 \%$ and $0 \%$. The weight percentages of $\mathrm{BP}$ were $0.5 \%$ and $0.1 \%$. The weight percentages of $\mathrm{NaCl}$ were $30 \%$ and $0 \%$. The two levels for the factor called bone fiber type are DMF and MBF. High and low levels were then combined according to the resolution III design to create eight formulations (Table 1(b)). This factorial design will demonstrates the effect that each parameter exhibits while minimizing the numbers of trials. The results from each experiment were examined to determine the main effects of each parameter on the measured property [18].

2.5. Scaffold Preparation. The designated amount of monomer (NVP) was divided into two portions. Threefourths of the monomer was combined with the PPF. The initiator (BP) was dissolved in the remaining one-fourth of the monomer and added to the polymer solution. The solid phase components $(\mathrm{BF}, \mathrm{NaCl})$ were added, followed by DMT. The resulting paste was immediately placed in 8-mm diameter glass vials. The resulting PPF cylinders (dry samples) were removed from the vials after overnight 


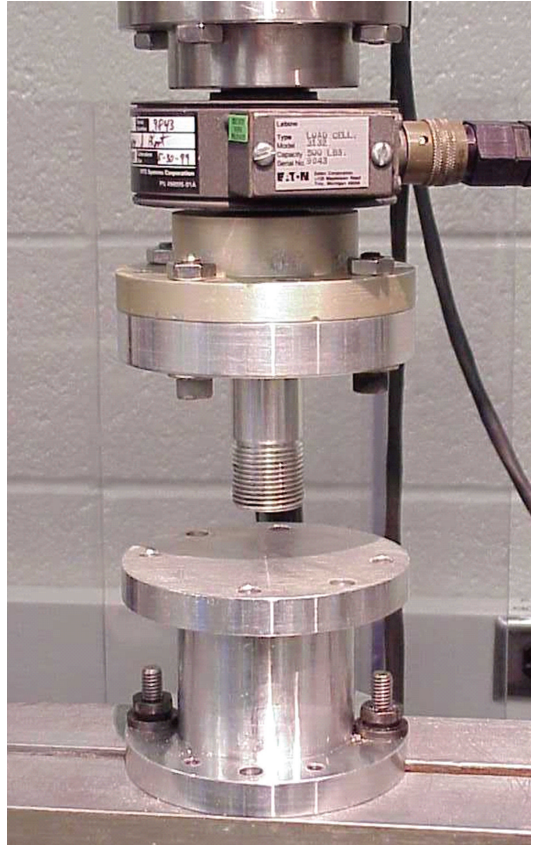

(a)

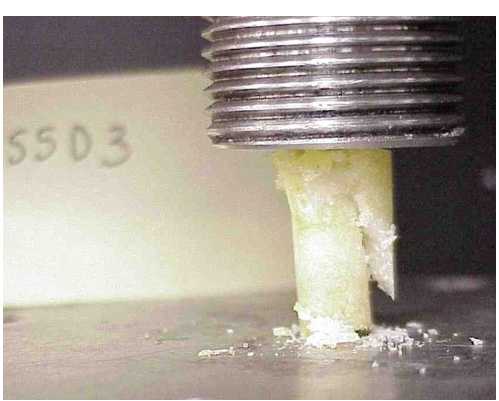

(b)

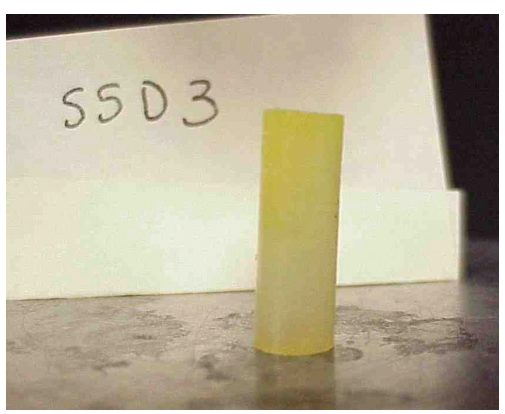

(c)

FIGURE 1: (a) The servohydraulic materials testing machine used in this study. (b) A representative PPF composite scaffold incorporating mineralized bone fibers and sodium chloride porogen. The PPF cylinder was approximately $6 \mathrm{~mm}$ in diameter and $20 \mathrm{~mm}$ in height. (c) The specimen was placed between two solid platens and compressed at a rate of $0.1 \mathrm{~mm} / \mathrm{second}$.

TABLE 1

(a) High and Low Levels for Six Parameters Tested in a Resolution III, Two-level Fractional Factorial Design

\begin{tabular}{|c|c|c|c|c|c|c|}
\hline Level & PPF Mn & NVP/PPF & BF/polymer & BF Type & $\mathrm{BP} / \mathrm{PPF}$ & $\mathrm{NaCl}$ \\
\hline+ & 4000 & $0.7 \mathrm{ml} / \mathrm{g}$ & $2 / 8$ & DBF & $0.5 \%$ & $30 \%$ \\
\hline- & 2000 & $0.5 \mathrm{ml} / \mathrm{g}$ & 0 & MBF & $0.1 \%$ & 0 \\
\hline
\end{tabular}

(b) Combinations of the Experimental Variables in the Resolution III, Two-level Fractional Factorial Design

\begin{tabular}{|c|c|c|c|c|c|c|}
\hline Formula & PPF Mn & NVP/PPF & $\mathrm{BF} /$ polymer & BF Type & $\mathrm{BP} / \mathrm{PPF}$ & $\mathrm{NaCl}$ \\
\hline 1 & + & + & + & + & + & + \\
\hline 2 & + & + & - & none & - & - \\
\hline 3 & + & - & + & - & + & - \\
\hline 4 & + & - & - & none & - & + \\
\hline 5 & - & + & + & - & - & + \\
\hline 6 & - & + & - & none & + & - \\
\hline 7 & - & - & + & + & - & - \\
\hline 8 & - & - & - & none & + & + \\
\hline
\end{tabular}

crosslinking. Half of the samples were incubated with phosphate buffered saline (PBS) for one day to leach out some of sodium chloride (wet samples). The scaffolds tested in this study had a diameter of $6 \mathrm{~mm}$ and a height of $20 \mathrm{~mm}$.

2.6. Mechanical Testing. Mechanical properties of both dry and wet samples were analyzed using a servohydraulic testing machine (MTS, Minneapolis, MN), as shown in Figure 1 (a). Sample length and diameter were measured before testing (Figure 1 (b)). The specimens were placed between two solid platens and compressed at a rate of $0.1 \mathrm{~mm} / \mathrm{second}$
(Figure $1(\mathrm{c})$ ). Load and displacement were recorded using a digital computer (Figure 2). The stress-strain curve was plotted by determining stress as the load divided by the cross-sectional area and strain as the displacement divided by the initial length of the cylinder. Compressive modulus was calculated as the slope of the initial linear portion of the stress-strain curve, beginning at $1.0 \%$ strain. The highest strength achieved was the ultimate strength.

2.7. Statistical Analysis. All data are reported as means \pm standard deviations (SDs) for $n=4$, except for size 
TABLE 2: Summary of Results for Ultimate Strength and Compressive Modulus.

\begin{tabular}{lcccc}
\hline \multicolumn{2}{c}{ Dry Samples } & & \multicolumn{2}{c}{ Wet Samples } \\
\hline Formulation & Modulus (MPa) & Ultimate Strength (MPa) & Modulus (MPa) & Ultimate Strength (MPa) \\
\hline 1 & $117 \pm 39$ & $9.9 \pm 0.3$ & $24.5 \pm 4.6$ & $3.3 \pm 0.4$ \\
2 & $108 \pm 15$ & $13.2 \pm 14$ & $66.0 \pm 7.7$ & $7.0 \pm 0.7$ \\
3 & $246 \pm 1.0$ & $11.1 \pm 0.6$ & $358 \pm 5.2$ & $16.6 \pm 4.4$ \\
4 & $271 \pm 29$ & $20.3 \pm 8.0$ & $184 \pm 1.3$ & $12.0 \pm 0.5$ \\
5 & $258 \pm 25$ & $13.3 \pm 1.0$ & $157 \pm 22$ & $8.7 \pm 2.1$ \\
6 & $184 \pm 0.5$ & $13.0 \pm 0.5$ & $101 \pm 0.2$ & $9.2 \pm 0.6$ \\
7 & $21.3 \pm 5.7$ & $2.1 \pm 0.9$ & $2.8 \pm 0.1$ & $0.4 \pm 0.0$ \\
8 & $220 \pm 17$ & $18.4 \pm 4.0$ & $157 \pm 8.2$ & $11.0 \pm 2.0$ \\
\hline
\end{tabular}

Values are given as means \pm standard deviations for $n=4$.

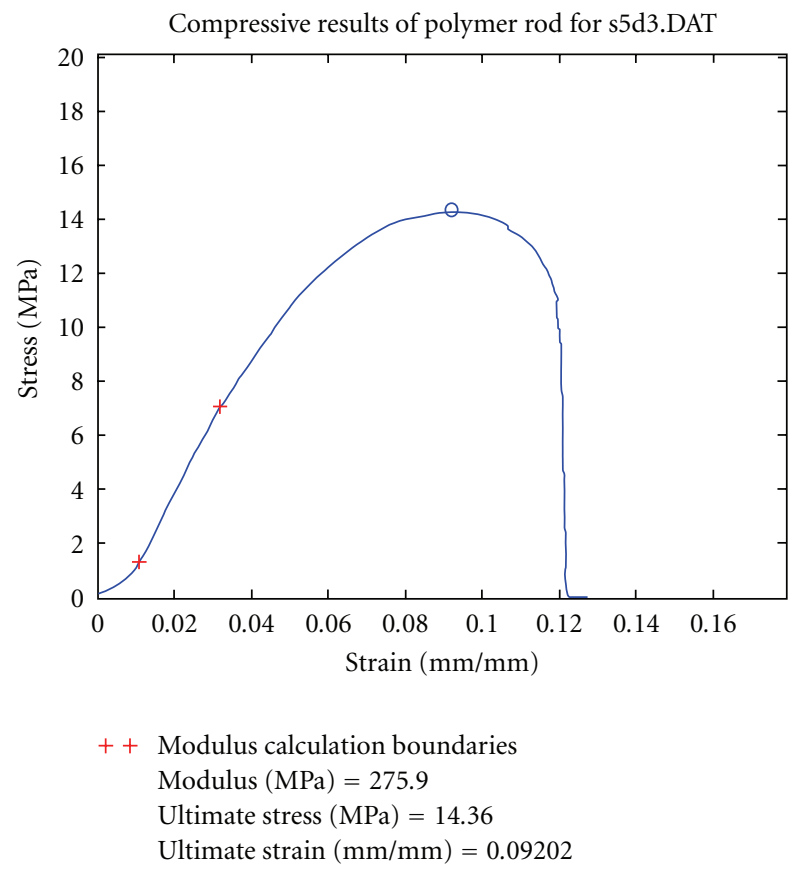

Figure 2: Load and displacement of the representative sample shown in Figure 1 were recorded during compression. The stressstrain curve was then plotted and compressive modulus was calculated as the slope of the initial linear portion of the stress-strain curve, beginning at $1.0 \%$ strain. The highest strength achieved was the ultimate strength.

distribution measurements where SD was calculated based on normal distribution. Single factor analysis of variance (ANOVA) was used to assess the statistical significance of results. Scheffés method was employed for multiple comparison tests at significance levels of 95 and 99\%.

\section{Results}

This study was designed to determine the effects of six parameters on the ultimate stress and compressive modulus of PPF/bone fiber composite scaffolds. The six parameters included PPF molecular weight, NVP to PPF ratio, BF to

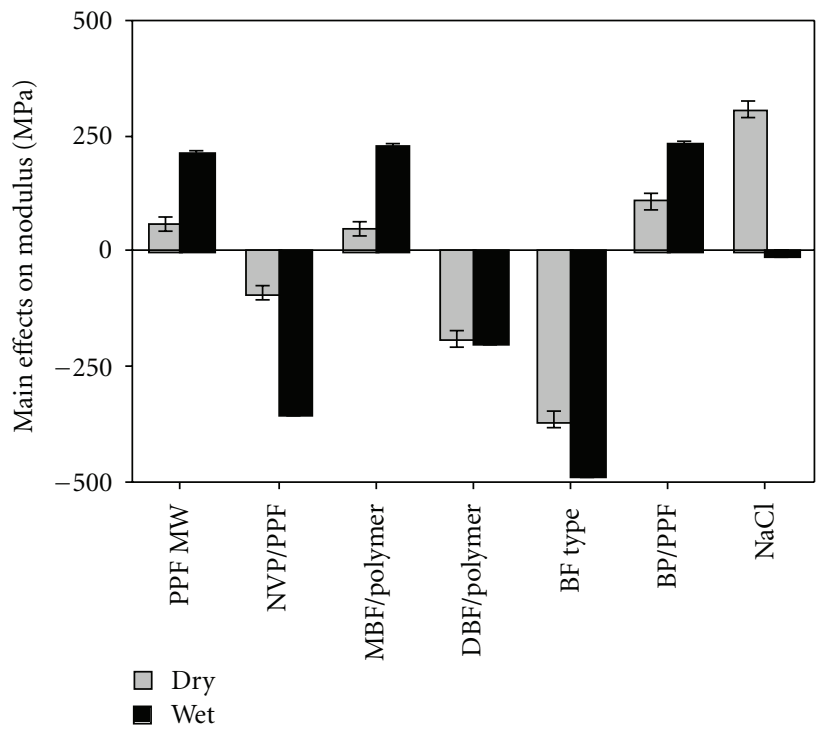

FIGURE 3: The main effects of the parameters on the compressive modulus of the crosslinked PPF composites. A positive number indicates an increase in the modulus as the parameter was changed from a low ( - ) level to a high $(+)$ level. A negative number indicates a decrease in the modulus as the parameter was changed from a low $(-)$ level to a high $(+)$ level. Error bars represent the standard error of the effect (dry $\pm 16.5 \mathrm{MPa}$, wet $\pm 6.2 \mathrm{MPa})$.

polymer (PPF/NVP) ratio, $\mathrm{BF}$ type, $\mathrm{BP}$ to $\mathrm{PPF}$ ratio, and the percentage of $\mathrm{NaCl}$ in the composites. The ultimate stress and compressive modulus of all formulations were measured and values are given in Table 2.

3.1. Compressive Modulus. Absolute values of compressive moduli varied from 21.3 to $271 \mathrm{MPa}$ and 2.8 to $358 \mathrm{MPa}$ for dry and wet samples, respectively (Table 2 ). The results from each formulation were calculated to determine the effects of each parameter on the measured property (Figure 3). In dry samples, the presence of $\mathrm{NaCl}$ significantly increased the observed modulus values and overshadowed the effects contributed by other factors. This increased modulus was not seen in the corresponding wet samples. Therefore, the mechanical data from wet samples are more suitable for 


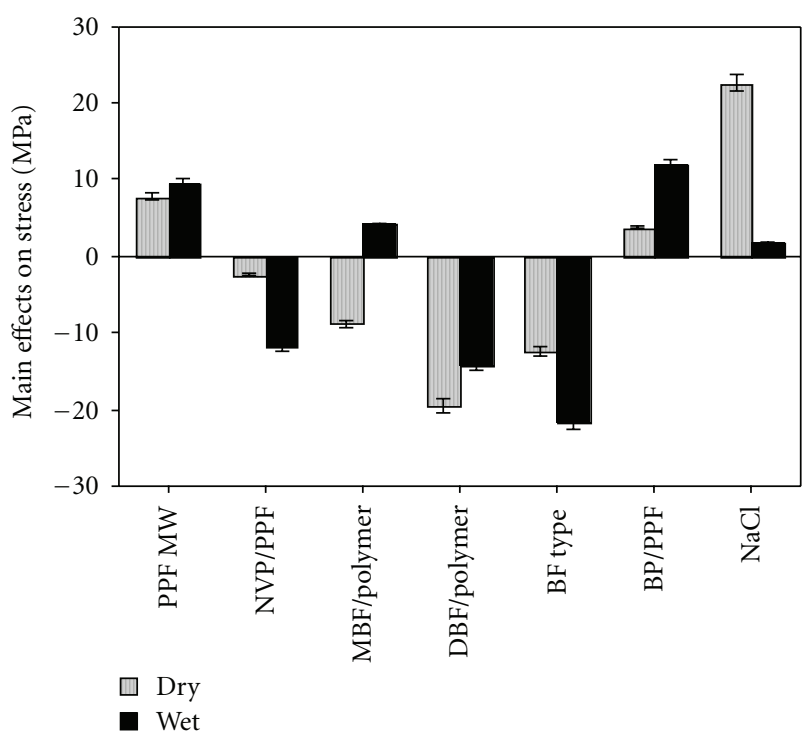

Figure 4: The main effects of the parameters on the ultimate strength of the crosslinked PPF composites. A positive number indicates an increase in the strength as the parameter was changed from a low ( -$)$ level to a high $(+)$ level. A negative number indicates a decrease in the strength as the parameter was changed from a low $(-)$ level to a high $(+)$ level. Error bars represent the standard error of the effect (dry $\pm 16.5 \mathrm{MPa}$, wet $\pm 6.2 \mathrm{MPa})$.

analysis of other effects. The incorporation of mineralized bone fibers and the increase of PPF molecular weight significantly increased the compressive modulus of PPF/NVP scaffolds. A decrease in monomer (NVP) led to an increase in compressive modulus. An increase in radical initiator (BP) was also found to increase the compressive modulus. Demineralization of bone fibers significantly decreased the compressive modulus of the scaffolds.

3.2. Ultimate Strength. The ultimate strength of the crosslinked composites varied from 2.1 to $20.3 \mathrm{MPa}$ for dry samples and from 0.4 to $16.6 \mathrm{MPa}$ for wet samples (Table 2). Very similar to compressive modulus, the presence of $\mathrm{NaCl}$ in dry samples significantly increased the observed modulus values and overshadowed the effects contributed by other factors (Figure 4), a phenomena not observed in the corresponding wet samples. The incorporation of MBF and the increase of PPF molecular weight were found to increase the ultimate strength of PPF/NVP scaffolds dramatically. Ultimate strength also increased as the amount of benzoyl peroxide increased or the amount of NVP decreased in the composites. Demineralization of bone fibers significantly decreased the ultimate strength of the scaffolds.

\section{Discussion}

Biodegradable PPF is a promising orthopedic material that may be used as injectable or preformed scaffolds to repair and regenerate bone defects [19-21]. The components used to form PPF scaffolds have a large influence on their mechanical properties. The objective of our study was to measure the compressive modulus and ultimate strength to determine the effects of the incorporation of bone fibers, PPF molecular weight, NVP amount, BP amount, $\mathrm{NaCl}$ amount, and demineralization of bone fibers on these measured properties. It was the first time that the effects of the incorporation of bone fibers and demineralization of bone fibers on PPF scaffolds were investigated.

New and important factors have been identified to affect the mechanical properties of PPF/NVP scaffolds. The incorporation of mineralized bone fibers and an increase in PPF molecular weight resulted in higher compressive modulus and ultimate strength. Both mechanical properties also increased when the amount of benzoyl peroxide increased or the amount of NVP decreased. Sodium chloride had a dominating effect on the increase of mechanical properties in dry samples but showed little effects in wet samples. Demineralized bone fibers led to a decrease in the compressive modulus and ultimate strength.

Our results are consistent with a previous finding that compressive modulus and ultimate strength increased with increasing the amount of benzoyl peroxide and a decrease in NVP amount [13]. PPF molecular weight was also found to have great influence on the mechanical properties. At higher molecular weights (2000 to 5000), it was reported that PPF molecular weight did not affect the mechanical strength [13]. This result, however, was based on dry samples incorporating $\mathrm{NaCl}$ to determine the main effects. As shown in our study, the dominating effect of $\mathrm{NaCl}$ in dry samples masked the effects of other factors. Therefore, wet samples are more appropriate to determine the effects of factors other than $\mathrm{NaCl}$.

We demonstrated for the first time that the incorporation of mineralized bone fibers in PPF/NVP scaffolds increases the mechanical properties significantly and in contrast, the incorporation of demineralized bone fibers decrease the mechanical properties significantly. Considering the fact that both bone fibers are biodegradable and DMF are osteoinductive, our results showed that $\mathrm{PPF} / \mathrm{bone}$ fiber composites with a wide range of mechanical properties could be fabricated for different clinical uses.

In summary, we have identified the important factors that affect the compressive modulus and ultimate strength of PPF/bone fiber scaffolds. The desired orthopedic PPF scaffold might be obtained by varying these factors. Our results suggest that bone fibers are appropriate as structural enforcement components in PPF scaffolds, and may modulate other aspects of the synthetic biomaterials such as osteoconductivity.

\section{Acknowledgments}

This paper was supported by the John Smith Foundation, Mayo Foundation, and National Institutes of Health (R01 EB30005 and AR 45871). 


\section{References}

[1] L. Lu, B. L. Currier, and M. J. Yaszemski, "Synthetic bone substitutes," Current Opinion in Orthopaedics, vol. 11, no. 5, pp. 383-390, 2000.

[2] A. G. Mikos, L. Lu, J. S. Temenoff, and J. K. Tessmar, "Synthetic bioresorbable polymer scaffolds," in Biomaterials Science, B. D. Ratner, A. S. Hoffman, F. J. Schoen, and J. E. Lemons, Eds., pp. 735-749, Elsevier, New York, NY, USA, 2nd edition, 2004.

[3] L. Lu, E. Jabbari, M. J. Moore, and M. J. Yaszemski, "Animal models for evaluation of tissue engineered orthopedic implants," in The Biomedical Engineering Handbook, J. D. Bronzino, Ed., chapter 45, pp. 1-10, CRC Press, Boca Raton, Fla, USA, 3rd edition, 2006.

[4] S. Wang, L. Lu, B. L. Currier, and M. J. Yaszemski, "Orthopedic prosthesis and joint implants," in An Introduction to Biomaterials, S. A. Guelcher and J. O. Hollinger, Eds., Biomedical Engineering Series, pp. 369-393, CRC / Taylor \& Francis, Boca Raton, Fla, USA, 2006.

[5] M. Light and I. O. Kanat, "The possible use of coralline hydroxyapatite as a bone implant," Journal of Foot Surgery, vol. 30, no. 5, pp. 472-476, 1991.

[6] S. Wang, L. Lu, and M. J. Yaszemski, "Bone-tissueengineering material poly(propylene fumarate): correlation between molecular weight, chain dimension, and physical properties," Biomacromolecules, vol. 7, no. 6, pp. 1976-1982, 2006.

[7] K.-W. Lee, S. Wang, L. Lu, E. Jabbari, B. L. Currier, and M. J. Yaszemski, "Fabrication and characterization of poly(propylene fumarate) scaffolds with controlled pore structures using 3-dimensional printing and injection molding," Tissue Engineering, vol. 12, no. 10, pp. 2801-2811, 2006.

[8] K.-W. Lee, S. Wang, M. J. Yaszemski, and L. Lu, "Physical properties and cellular responses to crosslinkable poly(propylene fumarate)/hydroxyapatite nanocomposites," Biomaterials, vol. 29, no. 19, pp. 2839-2848, 2008.

[9] S. Wang, D. H. R. Kempen, M. J. Yaszemski, and L. Lu, "The roles of matrix polymer crystallinity and hydroxyapatite nanoparticles in modulating material properties of photo-crosslinked composites and bone marrow stromal cell responses," Biomaterials, vol. 30, no. 20, pp. 3359-3370, 2009.

[10] X. Zhu, L. Lu, N. Liu, P. Chu, B. L. Currier, and M. J. Yaszemski, "Mechanical properties of biodegradable poly(propylene fumarate/bone fiber composites," Transactions Society of Biomaterials, vol. 25, p. 260, 2002.

[11] S. He, J. Ulrich, R. G. Valenzuela, et al., "Mechanical properties of biodegradable poly(propylene fumarate)-bone fiber composites during the degradation process," Transactions Society of Biomaterials, vol. 24, p. 149, 2001.

[12] B. D. Porter, J. B. Oldham, S.-L. He et al., "Mechanical properties of a biodegradable bone regeneration scaffold," Journal of Biomechanical Engineering, vol. 122, no. 3, pp. 286288, 2000.

[13] S. Wang, L. Lu, J. A. Gruetzmacher, B. L. Currier, and M. J. Yaszemski, "A biodegradable and cross-linkable multiblock copolymer consisting of poly(propylene fumarate) and $\operatorname{poly}(\varepsilon$-caprolactone): synthesis, characterization, and physical properties," Macromolecules, vol. 38, no. 17, pp. 7358-7370, 2005.

[14] S. Wang, D. H. Kempen, N. K. Simha, et al., "Photocrosslinked hybrid polymer networks for bone and nerve tissue-engineering applications: controlled physical properties and regulated cell responses," Biomacromolecules, vol. 9, pp. 1229-1241, 2008.
[15] A. B. M. Rabie, R. W. K. Wong, and U. Hägg, "Composite autogenous bone and demineralized bone matrices used to repair defects in the parietal bone of rabbits," British Journal of Oral and Maxillofacial Surgery, vol. 38, no. 5, pp. 565-570, 2000.

[16] K. D. Chesmel, J. Branger, H. Wertheim, and N. Scarborough, "Healing response to various forms of human demineralized bone matrix in athymic rat cranial defects," Journal of Oral and Maxillofacial Surgery, vol. 56, no. 7, pp. 857-865, 1998.

[17] S. J. Peter, L. J. Suggs, M. J. Yaszemski, P. S. Engel, and A. G. Mikos, "Synthesis of poly(propylene fumarate) by acylation of propylene glycol in the presence of a proton scavenger," Journal of Biomaterials Science, Polymer Edition, vol. 10, no. 3, pp. 363373, 1999.

[18] G. Box, W. Hunter, and J. S. Hunter, Statistics for Experimenters, John Wiley \& Sons, New York, NY, USA, 1978.

[19] D. H. R. Kempen, M. C. Kruyt, L. Lu et al., "Effect of autologous bone marrow stromal cell seeding and bone morphogenetic protein-2 delivery on ectopic bone formation in a microsphere/poly(propylene fumarate) composite," Tissue Engineering, Part A, vol. 15, no. 3, pp. 587-594, 2009.

[20] D. H. R. Kempen, M. J. Yaszemski, A. Heijink et al., "Noninvasive monitoring of BMP-2 retention and bone formation in composites for bone tissue engineering using SPECT/CT and scintillation probes," Journal of Controlled Release, vol. 134, no. 3, pp. 169-176, 2009.

[21] D. H. R. Kempen, L. Lu, A. Heijink et al., "Effect of local sequential VEGF and BMP-2 delivery on ectopic and orthotopic bone regeneration," Biomaterials, vol. 30, no. 14, pp. 2816-2825, 2009. 

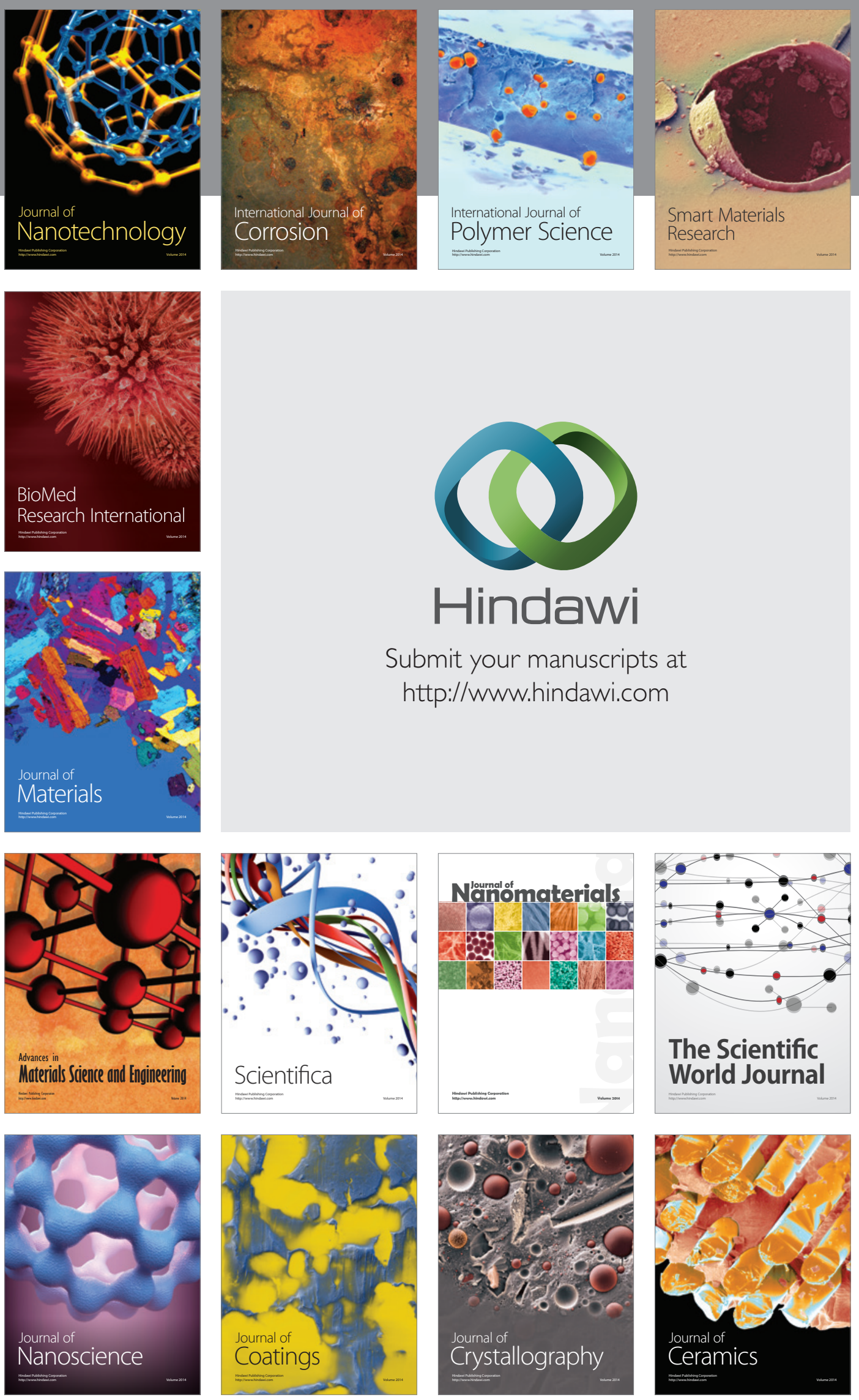

The Scientific World Journal

Submit your manuscripts at

http://www.hindawi.com

\section{World Journal}

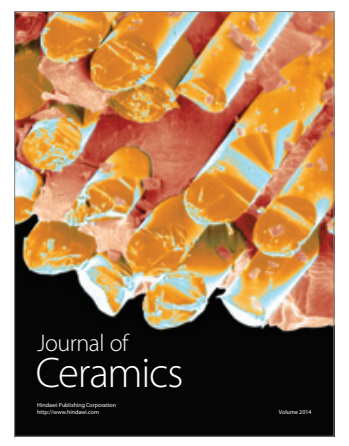

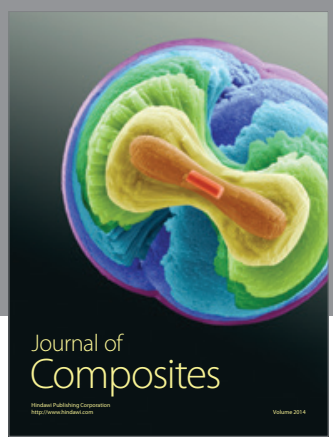
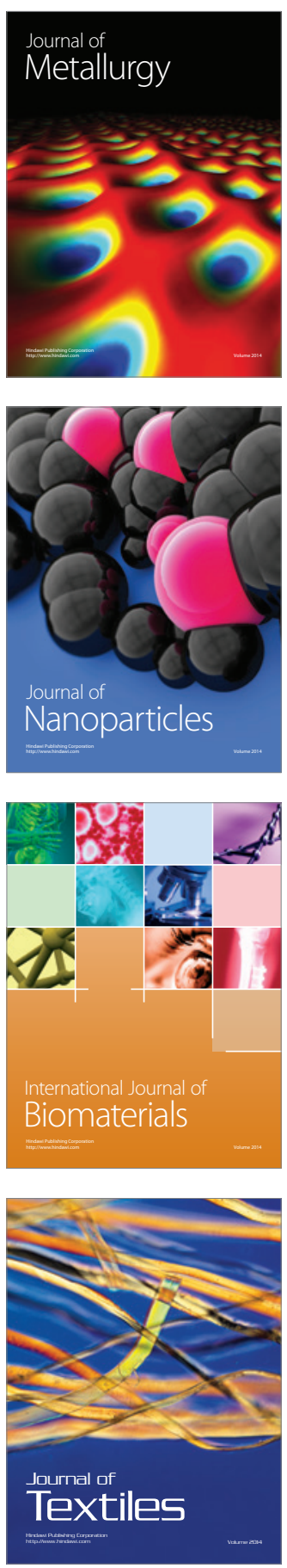